\title{
Association of tobacco and marijuana use with symptoms of depression and anxiety among adolescents and young adults in Texas
}

\author{
Aslesha Sumbe', Anna V. Wilkinson', Stephanie L. Clendennen', Bara S. Bataineh², Kymberle L. Sterling³, Baojiang \\ Chen ${ }^{4}$, Melissa B. Harrell
}

\begin{abstract}
INTRODUCTION Young people with depression and/or anxiety may self-medicate with tobacco or tobacco with marijuana to reduce their symptoms. This study sought to differentiate between the use of tobacco products as intended and tobacco products modified to accommodate marijuana, and to explore their relationships with symptoms of depression and anxiety among youth and young adults.

METHOdS The study is a secondary analysis of Wave 9 (Spring 2019) data from the Texas Adolescent Tobacco and Marketing Surveillance (TATAMS) ( $\mathrm{n}=2439$, $\mathrm{N}=274030$ ). Anxiety and major depressive symptoms were measured by GAD-7 and PHQ-9, respectively.

RESULTS The odds of reporting current use of e-cigarettes without marijuana (adjusted prevalence odds ratio, APOR $=2.34$; 95\% CI: $1.30-4.21, \mathrm{p}=0.005$ ) and current use of combustible tobacco without marijuana (APOR $=2.99 ; 95 \%$ CI: $1.26-7.09, \mathrm{p}=0.014)$ were significantly higher among those who reported depression/anxiety comorbidity compared to those who reported no symptoms of major depressive symptoms (MDS), anxiety or comorbidity. The odds of reporting ever use of e-cigarettes with marijuana (APOR $=3.68$; 95\% CI: 1.69 $8.00, \mathrm{p}=0.001$ ), current use of e-cigarettes with marijuana (APOR $=2.76 ; 95 \%$ CI: $1.28-5.97, \mathrm{p}=0.01$ ) and ever use of combustible tobacco with marijuana $(\mathrm{APOR}=3.99$; 95\% CI: 1.66-9.58, $\mathrm{p}=0.002)$ were significantly higher among those reporting only MDS compared to those who reported no symptoms of MDS, anxiety or comorbidity.

CONCLUSIONS The study findings can have implications for intervention planning, as interventions need to address marijuana and nicotine use in tobacco products and address anxiety and depression.
\end{abstract}

\section{AFFILIATION \\ 1 Department of Epidemiology, Human Genetics and Environmental Sciences, School of Public Health, University of Texas Health Science Center, Austin, United States \\ 2 Department of Epidemiology, Human Genetics and Environmental Sciences, School of Public Health, University of Texas Health Science Center, Dallas, United States \\ 3 Department of Health Promotion and Behavioral Sciences, School of Public Health, University of Texas Health Science Center, Dallas, United States \\ 4 Department of Biostatistics and Data Science, School of Public Health, University of Texas Health Science Center, Austin, United States}

CORRESPONDENCE TO

Aslesha Sumbe. Department of Epidemiology, Human Genetics and Environmental Sciences, School of Public Health, University of Texas Health Science Center, Austin, Texas 78701, United States. E-mail: aslesha.sumbe@uth.tmc.edu ORCID ID: https://orcid.org/00000002-6623-8543

\section{KEYWORDS}

adolescent, anxiety, depression, tobacco, young adult, marijuana

Received: 21 September 2021 Revised: 29 November 2021

Accepted: 3 December 2021

\section{INTRODUCTION}

Depression is a common mood or affective disorder, generally characterized by feelings of sadness and hopelessness, and is a significant cause of disability and addition to the burden of disease in the US.
The prevalence of major depressive episodes among US adolescents (aged 12-17 years) increased from $9.0 \%$ in 2004 to $13.3 \%$ in $2017^{1}$. Depression has high comorbidity with anxiety since $50-70 \%$ of people who report a lifetime history of a major depressive disorder 
also report a lifetime history of an anxiety disorder ${ }^{2}$. Anxiety disorder is characterized by the presence of extreme worry, anxiety or fear that is significant enough to interfere with daily life.

The Tripartite Model of anxiety and depression explains this comorbidity by splitting the symptoms of anxiety and depression into three dimensions: low positive affect, negative affect, and hyperarousal ${ }^{3}$. Negative affect is a characteristic shared by both depression and anxiety, which can explain the comorbidity. Low positive affect is a major component of depression, while hyperarousal is a major component of anxiety. Anxiety can also be a possible risk factor for depression. Some studies have found anxiety to occur before depression ${ }^{4,5}$, and comorbidity of anxiety among depressed youth to be more common than comorbidity of depression among youth with anxiety ${ }^{6}$. Although there is no causal relationship established, including anxiety in depression studies can be an important design factor.

Current epidemiologic literature shows depression and smoking behavior are related among adolescents ${ }^{7}$, although there is conflicting evidence for its direction, with some studies suggesting a bidirectional association ${ }^{8,9}$, indicative of self-medication. Although cigarette smoking has seen a decline in recent years, e-cigarette use has seen a sharp increase since its introduction to the US markets in 2007. Some studies have found an association between e-cigarette use and depression ${ }^{10,11}$.

Studies examining relationships between depression, anxiety, and smoking under the Tripartite model framework are rare. An extensive review of literature on the tripartite model and smoking found all three dimensions to have an association with smoking status ${ }^{12}$. A limitation of these studies is their focus on cigarette or combustible tobacco use among adults, while studies examining e-cigarette use and adolescents are lacking. Moreover, these studies only consider using tobacco products 'as intended', while today, these tobacco products are often modified by adding other substances such as marijuana.

Marijuana is a commonly used substance with $15.4 \%$ lifetime use prevalence among adolescents aged $12-17$ years and $51.5 \%$ lifetime use prevalence among young adults aged $18-25$ years $^{13}$. Co-use of marijuana and tobacco (e.g. marijuana use in a tobacco product) has been increasing ${ }^{14-16}$. In a California-based cohort of adolescents transitioning into young adulthood, co-use of cigarettes and smokeless tobacco with marijuana increased from $0.3 \%$ to $9.5 \%$ from 2008 to $2018^{17}$. Data from wave 2 (2014-2015) of the Population Assessment of Tobacco and Health (PATH) reported prevalence of co-use of tobacco and marijuana to be $21.3 \%$ among young adults aged 18-24 years while data from wave 3 (2015-2016) showed that about $80.1 \%$ of ever users of marijuana reported co-use with a tobacco product/device ${ }^{18,19}$. Marijuana use frequency has been associated with anhedonia and anxious arousal symptoms ${ }^{20}$, but studies exploring the association with co-use and among adolescents are lacking.

THC (tetrahydrocannabinol) and nicotine both trigger dopamine release, low levels of which are related to anhedonia ${ }^{21,22}$. Dopamine has also been associated with anxiety as it is released into the brain regions associated with anxiety ${ }^{23}$, including the amygdala ${ }^{24}$. Along with serotonin, dopamine has also been found to play a physiological role in social anxiety disorders ${ }^{25}$. The independent neurological impact of nicotine and marijuana is extensively studied, and various mechanisms have been put forth ${ }^{26}$. However, there is very limited research examining the impact of combined use of marijuana and nicotine on brain structures ${ }^{27}$. Moreover, psychopathology in the case of co-use of marijuana and tobacco has conflicting evidence ${ }^{28,29}$.

Adolescents with depression and/or anxiety may self-medicate with nicotine ${ }^{8}$ and/or marijuana ${ }^{30}$. Although there is evidence of differences in mental health symptoms between conventional cigarette users and e-cigarette users ${ }^{31}$, there is limited research examining tobacco products that are not used as intended but are modified to smoke marijuana instead of tobacco. This study sought to differentiate between the use of tobacco products as intended or tobacco products modified to accommodate marijuana, and to explore their relationships with symptoms of depression and anxiety among youth and young adults.

\section{METHODS}

\section{Study design and participants}

This study is a cross-sectional analysis of data 
collected by the Texas Adolescent Tobacco and Marketing Surveillance System (TATAMS) in Spring 2019. Sampling weights were created at Wave 1 specifically for these data and applied to account for the complex survey design so that results generalize from the sample (n) back to the population $(\mathrm{N})$ from which they were drawn ${ }^{32}$. Participants of the study were adolescents and young adults living in Texas's major metropolitan areas (Houston, Dallas/ Ft. Worth, San Antonio, Austin). A full description of the design and sampling methods used in TATAMS is reported elsewhere ${ }^{32}$. In Spring 2019, a total of 2441 individuals participated in the survey. The eligible sample for analysis in this study included any participant who provided complete data on measures of symptoms of depression, symptoms of anxiety, and tobacco and marijuana use behaviors $(n=2439$, $\mathrm{N}=274030)$. Among these, $695(\mathrm{~N}=86868 ; 31.7 \%)$ were in the 10th grade, $826(\mathrm{~N}=92183 ; 33.6 \%)$ were in the 12th grade, and $918(\mathrm{~N}=94979 ; 34.7 \%)$ were two years post high school.

\section{Measures}

Tobacco use (use of tobacco products without marijuana) To assess the use of tobacco products without marijuana, participants were explicitly told 'marijuana does not count' when they were presented with the following questions.

\section{Ever e-cigarette use}

Ever e-cigarette use without marijuana was assessed by asking: 'Have you ever used an electronic cigarette, vape pen, or e-hookah, even one or two puffs?'. Those who responded 'yes' to this question were categorized as ever e-cigarette users.

\section{Current e-cigarette use}

Current e-cigarette use without marijuana was assessed by asking: 'During the past 30 days, did you use an electronic cigarette?'. Participants who responded 'yes' were categorized as current e-cigarette users.

\section{Ever combustible tobacco use}

Ever combustible tobacco use without marijuana was assessed by asking the following questions: 'Have you ever tried cigarette smoking, even one or two puffs?', 'Have you ever tried smoking a little filtered cigar or cigarillo, even one or two puffs?', 'Have you ever smoked a tobacco-filled large cigar, even one or two puffs?' and 'Have you ever smoked a hookah, even one or two puffs?'. Participants who responded 'yes' to any of these questions were categorized as ever combustible tobacco users.

\section{Gurrent combustible tobacco use}

Current combustible tobacco use without marijuana was assessed by asking the following questions: 'During the past 30 days, did you smoke a cigarette/ little filtered cigar or cigarillo/large cigar/ hookah?'. Participants who responded 'yes' were categorized as current combustible tobacco user without marijuana.

Tobacco with marijuana use (use of tobacco products with marijuana)

Ever e-cigarette use

Ever e-cigarette use with marijuana was assessed by asking: 'Have you ever smoked marijuana (liquid THC) from an electronic cigarette, even one or two puffs?'. Participants who responded 'yes' to this question were categorized as ever users of e-cigarette with marijuana.

\section{Current e-cigarette use}

Current e-cigarette use with marijuana was assessed by asking 'During the past 30 days, on how many days did you smoke marijuana (liquid THC) from an electronic cigarette?'. Participants who responded to this question with a number between 1-30 were categorized as current e-cigarette users with marijuana.

\section{Ever combustible tobacco use}

Ever combustible tobacco use with marijuana was assessed by asking the following questions: 'Have you ever smoked marijuana in a hand-rolled cigarette with tobacco/spliff, even one or two puffs?', 'Have you ever smoked a little filtered cigar or cigarillo with marijuana in it, even one or two puffs?', 'Have you ever smoked a large cigar with marijuana in it, even one or two puffs? (blunts)' and 'Have you ever smoked marijuana in a hookah with a hose such as the one pictured above, even one or two puffs?'. Participants who responded 'yes' to any one of these questions were categorized as ever combustible tobacco users with marijuana. 
Current combustible tobacco use

Current combustible tobacco use with marijuana was assessed by asking the following questions: 'During the past 30 days, on how many days did you smoke marijuana from a cigarette?', 'During the past 30 days, on how many days did you smoke a little filtered cigar or cigarillo where the tobacco was taken out and replaced with marijuana?', 'During the past 30 days, on how many days did you smoke a large cigar where someone has replaced the tobacco with marijuana?' and 'During the past 30 days, on how many days did you smoke marijuana from a hookah?'. Participants who responded 'yes' to any one of these questions were categorized as current combustible tobacco users with marijuana.

Participants who responded affirmatively to both 'Tobacco use' (Use of tobacco products without marijuana) and 'Tobacco with marijuana use' (Use of tobacco products with marijuana) questions were categorized as tobacco users with marijuana, such that 'Tobacco use' and 'Tobacco with marijuana use' categories were mutually exclusive. This was to clearly distinguish the effects of only tobacco use from the interactive effects of tobacco and marijuana use.

\section{Major depressive symptoms}

Major depressive symptoms were assessed with the Patient Health Questionnaire (PHQ-9) ${ }^{33}$. Participants who reported the presence of five or more of the nine symptoms listed on this questionnaire, with at least one symptom being: 1) loss of interest or pleasure, or 2) depressed mood, were categorized as having major depressive symptoms (MDS).

\section{Anxiety symptoms}

Anxiety symptoms were assessed with the General Anxiety Disorder (GAD-7) instrument ${ }^{34}$. A severity score index was calculated by adding the scores assigned to the response options ranging from ' $0=\mathrm{Not}$ at all' to ' $3=$ Nearly every day' on the seven items. The recommended cutoff of 10 was used to categorize the presence of symptoms of anxiety.

\section{Depression/anxiety comorbidity}

Using measures outlined above, participants classified as having both major depressive symptoms and anxiety symptoms were categorized as having depression/ anxiety comorbidity.

\section{Sociodemographic covariates}

Four demographic covariates - school grade, gender, race/ethnicity, and SES - were selected based on the previous literature and included in the adjusted models. Grade level (10th, 12th, two years post high school), gender (male and female), socioeconomic status (SES), and race/ethnicity, were included as covariates.

To assess ethnicity and race, participants were asked 'Are you Hispanic or Latino/a?' and 'What race or races do you consider yourself to be?', respectively. The response options (Hispanic, White, Black or African American, Asian, American Indian or Alaska Native, Native Hawaiian or Other Pacific Islander and Other) were used to derive a single measure of race/ethnicity: Hispanic, non-Hispanic White, non-Hispanic Black, multi-racial, or other race/ethnicity.

To assess SES, participants were asked: 'In terms of income, what best describes your family's standard of living in the home where you live most of the time?' and the response options provided were 'Very well off,' 'Living comfortably', 'Just getting by', 'Nearly poor' and 'Poor'. 'Very well off' was categorized as 'High SES.' 'Living comfortably' was categorized as 'Middle SES' and 'Just getting by' 'Nearly poor' and 'Poor' were combined to 'Low SES'.

\section{Statistical analysis}

Descriptive and analytical statistics were computed using STATA 15.1 (College Station, TX). All analyses were weighted to represent the whole population and account for the complex design.

Descriptive statistics (frequency, percentage) were computed to describe sociodemographic characteristics, tobacco and tobacco with marijuana use in the sample by MDS, anxiety symptoms, and comorbidity.

Unadjusted and adjusted logistic regression models were fitted to assess the association between tobacco and tobacco with marijuana use, separately, with MDS, anxiety symptoms, and comorbidity. In separate analyses, the absence of MDS, anxiety symptoms, or comorbidity served as the reference category. The covariates - school grade, gender, 
race/ethnicity, and SES - were included in all adjusted models. Given the cross-sectional design, prevalence odds ratios (POR) are reported in this study.

\section{RESULTS}

Table 1 describes the sociodemographic characteristics and substance use of the sample by MDS, anxiety symptoms, and depression/anxiety comorbidity. Among the total participants in this study $(\mathrm{n}=2439$, $\mathrm{N}=274030), 8.4 \%(\mathrm{n}=231, \mathrm{~N}=23003)$ reported only symptoms of anxiety, $2.8 \%(\mathrm{n}=71, \mathrm{~N}=7795)$ reported only symptoms of MDS, and $10.9 \%(n=244, N=29898)$ reported depression/anxiety comorbidity, while $77.7 \%$ of the sample $(n=1893, N=213334)$ reported no symptom of either. Almost half of the sample consisted of females $(50.9 \%)$ and $54.9 \%$ were Hispanics. The most common form of substance use in the sample was ever use of combustible tobacco with marijuana (34.3\%) (e.g. as 'spliffs' or 'blunts') followed by ever use of e-cigarettes with marijuana $(26.1 \%)$ and ever use of e-cigarettes without marijuana $(22.8 \%)$. The current use of marijuana in e-cigarettes $(15.2 \%)$ and combustible tobacco $(15.1 \%)$ was higher than the current use of e-cigarettes and combustible tobacco without marijuana ( $7.4 \%$ and $3.0 \%$, respectively).

Table 1. Characteristics of sample (sample $n=2439$; population $N=274030$ ) by symptoms of mental health status; the Texas Adolescent Tobacco and Marketing Surveillance study (TATAMS), Spring $2019^{\text {a }}$

\begin{tabular}{|c|c|c|c|c|c|c|c|c|c|c|}
\hline & \multicolumn{2}{|r|}{ Total } & \multicolumn{2}{|r|}{ None } & \multicolumn{2}{|c|}{ Only anxiety } & \multicolumn{2}{|c|}{ Only MDS } & \multicolumn{2}{|c|}{$\begin{array}{c}\text { Depression/anxiety } \\
\text { comorbidity }^{b}\end{array}$} \\
\hline & $n$ & $\%$ & $n$ & $\%$ & $n$ & $\%$ & $n$ & $\%$ & $n$ & $\%$ \\
\hline & $\mathbf{N}^{c}$ & $95 \% \mathrm{CI}^{c}$ & $\mathbf{N}$ & $95 \% \mathrm{CI}$ & $\mathbf{N}$ & $95 \% \mathrm{CI}$ & $\mathbf{N}$ & $95 \% \mathrm{CI}$ & $\mathbf{N}$ & $95 \%$ CI \\
\hline \multirow[t]{2}{*}{ Total } & 2439 & 100 & 1893 & 77.85 & 231 & 8.39 & 71 & 2.84 & 244 & 10.91 \\
\hline & 274030 & - & 213334 & $(75.25-80.24)$ & 23003 & $(6.91-10.16)$ & 7795 & $(1.95-4.14)$ & 29898 & (9.33-12.73) \\
\hline \multicolumn{11}{|l|}{ Gender } \\
\hline \multirow[t]{2}{*}{ Female } & 1401 & 50.95 & 1001 & 36.14 & 171 & 5.58 & 45 & 1.66 & 184 & 7.57 \\
\hline & 139618 & $(45.04-56.84)$ & 99034 & $(32.15-40.32)$ & 15302 & $(4.26-7.29)$ & 4549 & (1.04-2.64) & 20753 & $(6.25-9.15)$ \\
\hline \multirow[t]{2}{*}{ Male } & 1038 & 49.05 & 892 & 41.71 & 60 & 2.81 & 26 & 1.18 & 60 & 3.34 \\
\hline & 134412 & (43.16-54.96) & 114298 & (36.64-46.97) & 7700 & $(1.87-4.21)$ & 3242 & $(0.65-2.16)$ & 9152 & $(2.31-4.79)$ \\
\hline \multicolumn{11}{|l|}{ Grade } \\
\hline \multirow[t]{2}{*}{10 th } & 695 & 31.70 & 543 & 25.19 & 59 & 2.59 & 19 & 0.85 & 74 & 3.06 \\
\hline & 86868 & $(21.39-44.18)$ & 69028 & $(16.80-35.96)$ & 7108 & $(1.43-4.65)$ & 2330 & $(0.41-1.75)$ & 8402 & $(1.87-4.98)$ \\
\hline \multirow[t]{2}{*}{ 12th } & 826 & 33.64 & 656 & 26.54 & 80 & 2.73 & 25 & 1.23 & 65 & 3.14 \\
\hline & 92183 & $(23.1-46.11)$ & 72728 & (18.23-36.92) & 7479 & $(1.81-4.09)$ & 3368 & $(0.53-2.81)$ & 8618 & $(2.07-4.76)$ \\
\hline \multirow{2}{*}{$\begin{array}{l}2 \text { Years after } \\
\text { High School }\end{array}$} & 918 & 34.66 & 694 & 26.12 & 92 & 3.07 & 27 & 0.76 & 105 & 4.70 \\
\hline & 94979 & (23.44-47.88) & 71577 & (17.88-36.47) & 8415 & $(1.97-4.76)$ & 2094 & $(0.44-1.31)$ & 12883 & $(3.08-7.11)$ \\
\hline \multicolumn{11}{|l|}{ Race/ethnicity } \\
\hline \multirow{2}{*}{$\begin{array}{l}\text { Non-Hispanic } \\
\text { Black }\end{array}$} & 359 & 15.29 & 285 & 12.60 & 36 & 1.45 & 8 & 0.17 & 30 & 1.06 \\
\hline & 41899 & (11.93-19.38) & 34527 & $(9.74-16.16)$ & 3973 & $(0.89-2.37)$ & 478 & $(0.074-0.41)$ & 2921 & $(0.56-1.97)$ \\
\hline \multirow[t]{2}{*}{ Hispanic } & 915 & 54.88 & 689 & 41.59 & 77 & 4.18 & 30 & 1.81 & 119 & 7.31 \\
\hline & 150388 & (48.02-61.56) & 113969 & $(36.45-46.93)$ & 11447 & $(3.10-5.61)$ & 4949 & (1.09-2.99) & 20023 & (5.69-9.33) \\
\hline \multirow[t]{2}{*}{ Other ${ }^{d}$} & 390 & 11.25 & 311 & 8.82 & 34 & 0.97 & 14 & 0.45 & 31 & 1.01 \\
\hline & 30827 & (8.93-14.08) & 24169 & (6.86-11.28) & 2657 & $(0.58-1.63)$ & 1248 & $(0.20-1.01)$ & 2763 & $(0.65-1.56)$ \\
\hline \multirow{2}{*}{$\begin{array}{l}\text { Non-Hispanic } \\
\text { White }\end{array}$} & 775 & 18.57 & 608 & 14.83 & 84 & 1.79 & 19 & 0.41 & 64 & 1.53 \\
\hline & 50886 & $(13.65-24.76)$ & 40639 & $(10.80-20.05)$ & 4927 & $(1.28-2.53)$ & 1123 & $(0.22-0.73)$ & 4197 & $(0.99-2.34)$ \\
\hline
\end{tabular}


Table 1. Continued

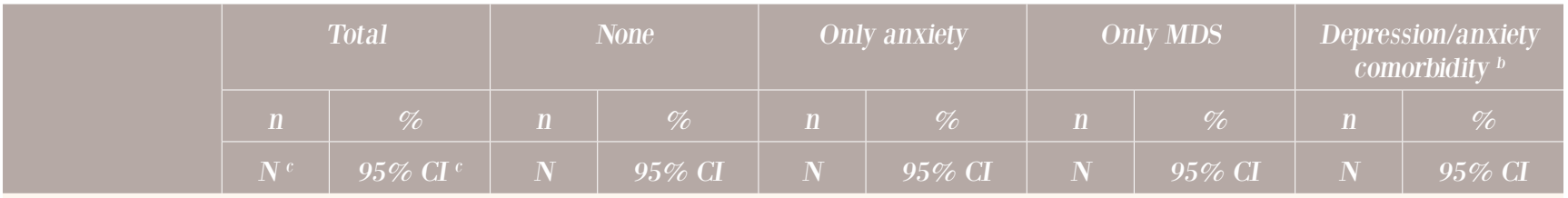

\section{Socioeconomic}

status $^{\mathrm{e}}$

$\begin{array}{lrcrcrrrrrrr}\text { High } & 299 & 10.37 & 251 & 8.93 & 23 & 0.62 & 5 & 0.18 & 20 & 0.65 \\ & 28415 & (7.20-14.73) & 24464 & (6.08-12.93) & 1689 & (0.36-1.06) & 488 & (0.05-0.61) & 1794 & (0.38-1.12) \\ \text { Middle } & 1539 & 63.34 & 1232 & 51.04 & 132 & 4.75 & 42 & 1.79 & 133 & 5.76 \\ & 173571 & (59.61-66.91) & 139863 & (48.08-53.98) & 13002 & (3.49-6.42) & 4912 & (1.16-2.76) & 15794 & (4.48-7.38) \\ \text { Low } & 601 & 26.28 & 410 & 17.89 & 76 & 3.03 & 24 & 0.87 & 91 & 4.49 \\ & 72014 & (22.55-30.40) & 49024 & (14.98-21.22) & 8309 & (2.05-4.47) & 2393 & (0.43-1.76) & 12318 & (3.35-6.00)\end{array}$

Tobacco use ${ }^{f}$

\begin{tabular}{|c|c|c|c|c|c|c|c|c|c|c|}
\hline \multirow[t]{2}{*}{ Ever e-cigarette } & 557 & 22.78 & 425 & 17.22 & 42 & 1.97 & 21 & 0.65 & 69 & 2.94 \\
\hline & 62406 & (19.09-26.94) & 47188 & $(14.72-20.06)$ & 5385 & $(1.24-3.11)$ & 1777 & $(0.36-1.18)$ & 8056 & $(1.97-4.37)$ \\
\hline \multirow{2}{*}{$\begin{array}{l}\text { Current } \\
\text { e-cigarette }\end{array}$} & 209 & 7.42 & 147 & 5.00 & 22 & 0.81 & 6 & 0.16 & 34 & 1.44 \\
\hline & 20328 & $(5.89-9.30)$ & 13710 & $(3.85-6.48)$ & 2220 & $(0.41-1.59)$ & 446 & $(0.04-0.59)$ & 3952 & $(0.86-2.41)$ \\
\hline \multirow[t]{2}{*}{ Ever combustible } & 250 & 10.66 & 194 & 7.91 & 23 & 1.49 & 7 & 0.18 & 26 & 1.08 \\
\hline & 29224 & $(8.75-12.94)$ & 21676 & $(6.01-10.34)$ & 4091 & $(0.79-2.78)$ & 495 & $(0.08-0.44)$ & 2962 & $(0.62-1.89)$ \\
\hline \multirow{2}{*}{$\begin{array}{l}\text { Current } \\
\text { combustible }\end{array}$} & 88 & 2.97 & 64 & 1.85 & 7 & 0.35 & 5 & 0.08 & 12 & 0.68 \\
\hline & 8131 & $(2.05-4.29)$ & 5072 & $(1.30-2.63)$ & 974 & $(0.11-1.16)$ & 213 & $(0.02-0.26)$ & 1871 & $(0.32-1.45)$ \\
\hline \multicolumn{11}{|l|}{$\begin{array}{l}\text { Tobacco with } \\
\text { marijuana use }\end{array}$} \\
\hline \multirow[t]{2}{*}{ Ever e-cigarette } & 635 & 26.10 & 466 & 18.94 & 66 & 2.33 & 29 & 1.53 & 74 & 3.29 \\
\hline & 71497 & (23.34-29.05) & 51901 & (16.49-21.67) & 6382 & $(1.56-3.46)$ & 4193 & $(0.85-2.76)$ & 9021 & $(2.45-4.42)$ \\
\hline \multirow{2}{*}{$\begin{array}{l}\text { Current } \\
\text { e-cigarette }\end{array}$} & 348 & 15.20 & 254 & 11.09 & 35 & 1.46 & 16 & 0.85 & 43 & 1.80 \\
\hline & 41635 & $(13.16-17.48)$ & 30390 & $(9.363-13.1)$ & 3990 & $(0.85-2.49)$ & 2322 & $(0.41-1.74)$ & 4933 & $(1.26-2.57)$ \\
\hline \multirow[t]{2}{*}{ Ever combustible } & 806 & 34.33 & 588 & 24.55 & 70 & 2.58 & 35 & 1.80 & 113 & 5.39 \\
\hline & 94060 & (30.54-38.33) & 67274 & $(21.85-27.47)$ & 7064 & $(1.69-3.89)$ & 4935 & (0.99-3.27) & 14787 & $(4.11-7.05)$ \\
\hline \multirow{2}{*}{$\begin{array}{l}\text { Current } \\
\text { combustible }\end{array}$} & 343 & 15.05 & 246 & 11.12 & 29 & 1.21 & 17 & 0.58 & 51 & 2.14 \\
\hline & 41227 & $(12.96-17.41)$ & 30472 & (9.32-13.21) & 3310 & (0.74-1.97) & 1589 & $(0.32-1.05)$ & 5856 & $(1.53-2.98)$ \\
\hline
\end{tabular}

a Row adds to 100\%. b Depression/Anxiety Comorbidity: Participants who were classified as having both Major Depressive Symptoms and Anxiety Symptoms. c N = weighted sample. $\%=$ weighted proportion. $\mathrm{Cl}$ : confidence interval. TATAMS is a probability-based sample, so results generalize back to the larger population from which it was drawn. d Includes Asian, American Indian or Alaska Native, Native Hawaiian or Other Pacific Islander and Other (not listed). e 'Very well off' classified as 'High SES'; 'Living comfortably' and 'just getting by' classified as 'Middle SES'; and 'Nearly poor' and 'Poor' classified as 'Low SES'. f Tobacco use without marijuana. $g$ Tobacco product use with marijuana.

Table 2 presents the unadjusted and adjusted POR describing the association between tobacco use without marijuana with only anxiety symptoms, only MDS, and their comorbidity, respectively.

Tobacco use did not have any significant relationship with anxiety and major depressive symptoms alone, both in unadjusted and adjusted models. After adjusting for sociodemographic variables, the odds of reporting current use of e-cigarettes without marijuana $(\mathrm{APOR}=2.34$; 95\% CI: $1.30-4.21, \mathrm{p}=0.005$ ) and current use of combustible tobacco without marijuana 
(APOR $=2.99 ; 95 \%$ CI: $1.26-7.09, \mathrm{p}=0.014)$ were significantly higher among those who reported depression/anxiety comorbidity compared to those who reported no symptoms of MDS, anxiety or comorbidity.

Table 3 presents the unadjusted and adjusted POR describing the association between tobacco use with marijuana with only anxiety symptoms, only MDS, and their comorbidity, respectively.

Tobacco use with marijuana did not have any significant relationship with anxiety symptoms, both in unadjusted and adjusted models. After adjusting for sociodemographic variables, the odds of reporting ever use of e-cigarettes with marijuana (APOR=3.68; 95\% CI: 1.69-8.00, p=0.001), current use of e-cigarettes with marijuana $(\mathrm{APOR}=2.76 ; 95 \%$ CI: $1.28-5.97, \mathrm{p}=0.01)$ and ever use of combustible tobacco with marijuana $(\mathrm{APOR}=3.99 ; 95 \% \mathrm{CI}$ : $1.66-9.58, p=0.002$ ) were significantly higher among those reporting only MDS compared to those who reported no symptoms of MDS, anxiety or comorbidity. The odds of reporting ever use of combustible tobacco with marijuana $(\mathrm{APOR}=1.87$; 95\% CI: $1.17-2.98, \mathrm{p}=0.009$ ) were significantly

Table 2. Association of tobacco use with anxiety, major depressive and comorbid symptoms ( $\mathrm{n}=2439$; N=274030); The Texas Adolescent Tobacco and Marketing Surveillance study (TATAMS), Spring 2019

\begin{tabular}{|c|c|c|c|c|c|c|c|c|}
\hline & \multicolumn{4}{|c|}{ POR (95\% CI) } & \multicolumn{4}{|c|}{$\operatorname{APOR}^{a}(95 \%$ CI $)$} \\
\hline & $\begin{array}{c}\text { No } \\
\text { symptoms }\end{array}$ & $\begin{array}{l}\text { Anxiety } \\
\text { symptoms }\end{array}$ & $\begin{array}{c}\text { Major } \\
\text { depressive } \\
\text { symptoms }\end{array}$ & $\begin{array}{l}\text { Comorbid } \\
\text { symptoms }^{b}\end{array}$ & $\begin{array}{c}\text { No } \\
\text { symptoms }\end{array}$ & $\begin{array}{l}\text { Anxiety } \\
\text { symptoms }\end{array}$ & $\begin{array}{c}\text { Major } \\
\text { depressive } \\
\text { symptoms }\end{array}$ & $\begin{array}{l}\text { Comorbid } \\
\text { symptoms }^{b}\end{array}$ \\
\hline \multicolumn{9}{|l|}{ Tobacco use } \\
\hline Ever e-cigarette & 1 & $\begin{array}{c}1.08 \\
(0.65-1.78)\end{array}$ & $\begin{array}{c}1.04 \\
(0.56-1.94)\end{array}$ & $\begin{array}{c}1.30 \\
(0.85-1.97)\end{array}$ & 1 & $\begin{array}{c}1.01 \\
(0.63-1.62)\end{array}$ & $\begin{array}{c}1.02 \\
(0.56-1.84)\end{array}$ & $\begin{array}{c}1.19 \\
(0.79-1.79)\end{array}$ \\
\hline Current e-cigarette & 1 & $\begin{array}{c}1.55 \\
(0.70-3.44)\end{array}$ & $\begin{array}{c}0.98 \\
(0.25-3.85)\end{array}$ & $\begin{array}{c}2.22 \\
(1.28-3.84)^{*}\end{array}$ & 1 & $\begin{array}{c}1.57 \\
(0.68-3.61)\end{array}$ & $\begin{array}{c}0.91 \\
(0.22-3.75)\end{array}$ & $\begin{array}{c}2.34 \\
(1.30-4.21)^{*}\end{array}$ \\
\hline Ever combustible & 1 & $\begin{array}{c}1.91 \\
(0.76-4.81)\end{array}$ & $\begin{array}{c}0.60 \\
(0.23-1.56)\end{array}$ & $\begin{array}{c}0.97 \\
(0.43-2.18)\end{array}$ & 1 & $\begin{array}{c}1.95 \\
(0.81-4.66)\end{array}$ & $\begin{array}{c}0.61 \\
(0.23-1.62)\end{array}$ & $\begin{array}{c}0.98 \\
(0.45-2.14)\end{array}$ \\
\hline Current combustible & 1 & $\begin{array}{c}1.81 \\
(0.50-6.61)\end{array}$ & $\begin{array}{c}1.27 \\
(0.34-4.69)\end{array}$ & $\begin{array}{c}2.74 \\
(1.19-6.31)^{*}\end{array}$ & 1 & $\begin{array}{c}1.90 \\
(0.57-6.34)\end{array}$ & $\begin{array}{c}1.23 \\
(0.33-4.57)\end{array}$ & $\begin{array}{c}2.99 \\
(1.26-7.09)^{*}\end{array}$ \\
\hline
\end{tabular}

a Models adjusted for grade, gender, race/ethnicity and SES. b Depression/anxiety comorbidity. ${ }^{*} \mathrm{p}<0.05$. POR: prevalence odds ratio. APOR: adjusted prevalence odds ratio.

Table 3. Association of tobacco with marijuana use with anxiety, major depressive and comorbid symptoms ( $n=2439 ; \mathrm{N}=274030)$; The Texas Adolescent Tobacco and Marketing Surveillance study (TATAMS), Spring 2019

\begin{tabular}{|c|c|c|c|c|c|c|c|}
\multicolumn{4}{|c|}{ POR $(95 \%$ CI $)$} & \multicolumn{4}{c|}{ APOR $^{\text {a }}(95 \%$ CI $)$} \\
\hline $\begin{array}{c}\text { No } \\
\text { symptoms }\end{array}$ & $\begin{array}{c}\text { Anxiety } \\
\text { symptoms }\end{array}$ & $\begin{array}{c}\text { Major } \\
\text { depressive } \\
\text { symptoms }\end{array}$ & $\begin{array}{c}\text { Comorbid } \\
\text { symptoms }^{b}\end{array}$ & $\begin{array}{c}\text { No } \\
\text { symptoms }\end{array}$ & $\begin{array}{c}\text { Anxiety } \\
\text { symptoms }\end{array}$ & $\begin{array}{c}\text { Major } \\
\text { depressive } \\
\text { symptoms }\end{array}$ & $\begin{array}{c}\text { Comorbid } \\
\text { symptoms }^{b}\end{array}$ \\
\hline
\end{tabular}

\begin{tabular}{|c|c|c|c|c|c|c|c|c|}
\hline \multicolumn{9}{|l|}{$\begin{array}{l}\text { Tobacco with } \\
\text { marijuana use }\end{array}$} \\
\hline Ever e-cigarette & 1 & $\begin{array}{c}1.19 \\
(0.83-1.72)\end{array}$ & $\begin{array}{c}3.62 \\
(1.75-7.52)^{*}\end{array}$ & $\begin{array}{c}1.34 \\
(0.9-2.01)\end{array}$ & 1 & $\begin{array}{c}1.14 \\
(0.76-1.7)\end{array}$ & $\begin{array}{c}3.68 \\
(1.69-8.00)^{*}\end{array}$ & $\begin{array}{c}1.19 \\
(0.76-1.84)\end{array}$ \\
\hline Current e-cigarette & 1 & $\begin{array}{c}1.26 \\
(0.71-2.23)\end{array}$ & $\begin{array}{c}2.93 \\
(1.35-6.32)^{*}\end{array}$ & $\begin{array}{c}1.19 \\
(0.75-1.88)\end{array}$ & 1 & $\begin{array}{c}1.22 \\
(0.66-2.26)\end{array}$ & $\begin{array}{c}2.76 \\
(1.28-5.97)^{*}\end{array}$ & $\begin{array}{c}1.04 \\
(0.63-1.73)\end{array}$ \\
\hline Ever combustible & 1 & $\begin{array}{c}0.96 \\
(0.58-1.60)\end{array}$ & $\begin{array}{c}3.75 \\
(1.62-8.71)^{*}\end{array}$ & $\begin{array}{c}2.12 \\
(1.42-3.18)^{*}\end{array}$ & 1 & $\begin{array}{c}0.89 \\
(0.51-1.55)\end{array}$ & $\begin{array}{c}3.99 \\
(1.66-9.58)^{*}\end{array}$ & $\begin{array}{c}1.87 \\
(1.17-2.98)^{*}\end{array}$ \\
\hline Current combustible & 1 & $\begin{array}{c}1.01 \\
(0.57-1.78)\end{array}$ & $\begin{array}{c}1.73 \\
(0.87-3.43)\end{array}$ & $\begin{array}{c}1.46 \\
(0.94-2.27)\end{array}$ & 1 & $\begin{array}{c}0.92 \\
(0.50-1.69)\end{array}$ & $\begin{array}{c}1.60 \\
(0.80-3.23)\end{array}$ & $\begin{array}{c}1.21 \\
(0.73-2.01)\end{array}$ \\
\hline
\end{tabular}

a Models adjusted for grade, gender, race/ethnicity and SES. b Depression/anxiety comorbidity. ${ }^{*} p<0.05$. POR: prevalence odds ratio. APOR: adjusted prevalence odds ratio. 
Figure 1. Figurative representation of tripartite model and study findings

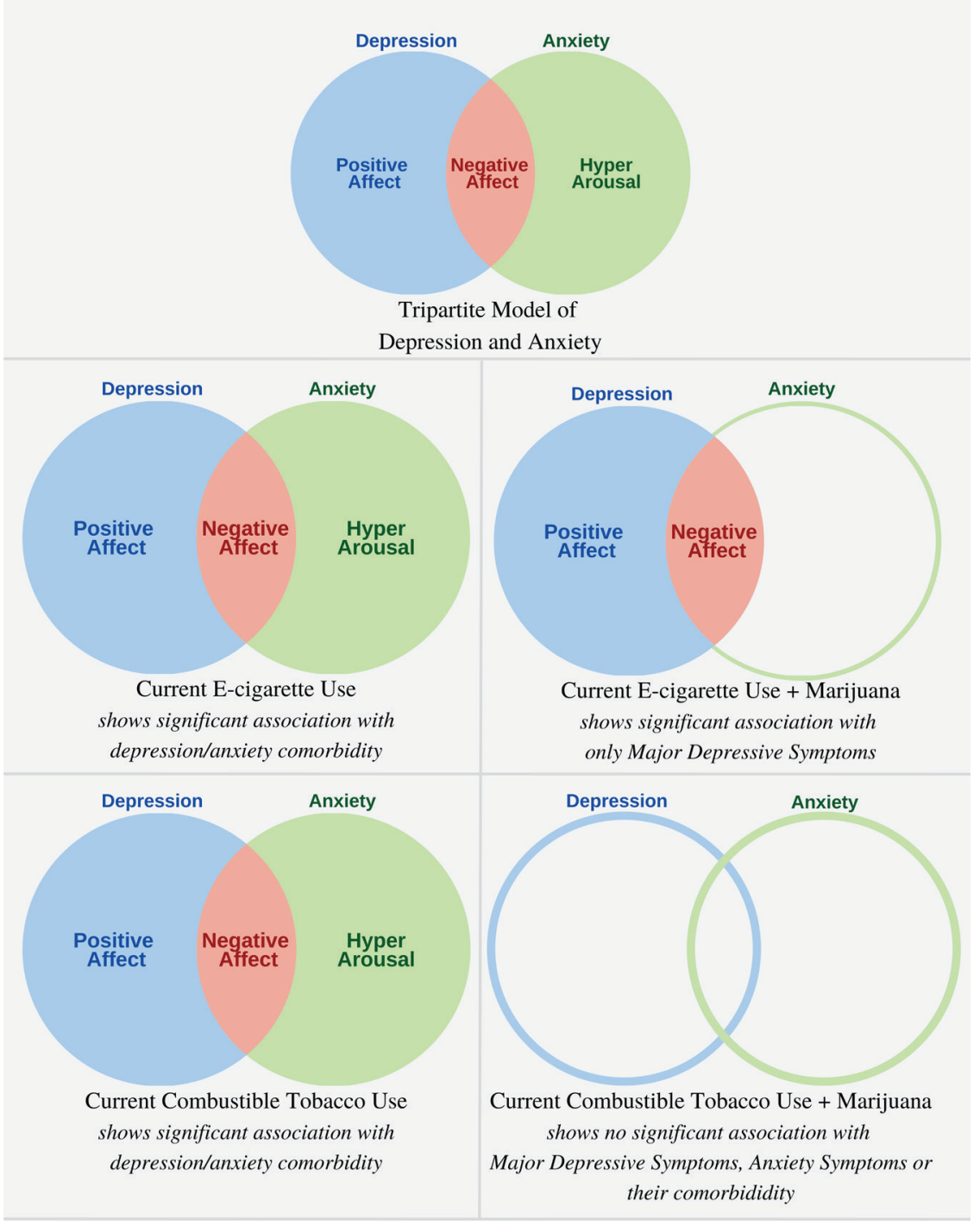

higher among those with a depression/anxiety comorbidity compared to those who reported no symptoms of MDS, anxiety or comorbidity.

\section{DISCUSSION}

We examined cross-sectional associations between tobacco used as intended and tobacco modified to smoke with marijuana and symptoms of depression, anxiety, and comorbid depression and anxiety. By separating tobacco use 'without marijuana' from 'with marijuana', we explored symptomatic differences by substance (i.e. nicotine only vs nicotine and marijuana). Given the unique characteristic of nicotine being both a stimulant and sedative at varying dosage, and marijuana being a stimulant and depressant as well, the relationships between nicotine, marijuana, depression and anxiety are complex ${ }^{29}$. The study made a preliminary attempt to dissect this web of interaction to obtain a snapshot of the phenomenon. Anxiety symptoms were not associated with tobacco use, with or without marijuana, while there were significant associations between tobacco (i.e. nicotine) use and symptoms of depression and comorbidity, which varied by the use of marijuana.

Ever use of tobacco reflects ever having experimented with a tobacco product. Previous 
research has shown that ever e-cigarette users reported lower levels of 'internalizing' mental health problems such as depression and anxiety syndrome than conventional cigarette users, although marijuana use was not examined ${ }^{31}$. In our study, neither ever use of either e-cigarettes or combustible tobacco products without marijuana were associated with anxiety. However, ever e-cigarette use with marijuana was associated with major depressive symptoms while ever combustible use was associated with comorbid symptoms of anxiety and major depressive symptoms.

Current use reflects product use at least once in the past 30 days. Here, e-cigarette use without marijuana was significantly associated with depression/anxiety comorbidity while e-cigarette use with marijuana was significantly associated with MDS only. Using the elements of Tripartite model, as described in Figure 1, e-cigarette use without marijuana shows significant association with low positive affect, high negative affect, and hyper arousal (depression/anxiety comorbidity), while e-cigarette use with marijuana shows significant association with only low positive affect and negative affect (MDS). This indicates that the addition of marijuana to e-cigarettes may have an impact on the hyperarousal dimension of anxiety. In a previous study, frequency of marijuana use, controlling for cigarette use, was associated with increased anxiety symptoms, and coping motives among youth mediated the association ${ }^{20}$. Hence, marijuana could be the compound used by youth to 'self-medicate' for anxiety, which might explain why, in our study, e-cigarette use alone is associated with depression/ anxiety comorbidity while e-cigarette use with marijuana is associated with MDS only.

Current use of combustible tobacco without marijuana was significantly associated with comorbid symptoms while current use of combustible tobacco with marijuana showed no significant associations. A previous study that examined the combined use of marijuana and cigarettes found that youth who reported only tobacco use had significantly higher negative affect than those who used marijuana and tobacco $^{29}$. The addition of marijuana to combustible tobacco suggests the possibility of quelling both depressive and anxiety symptoms, possibly impacting all three dimensions of the Tripartite model.

\section{Strengths and limitations}

The study has some limitations. The cross-sectional design limits our ability to examine temporality; we cannot determine if the symptoms are risk factors for smoking or a result of smoking. As we used the general recommended cutoff for anxiety to examine general anxiety symptoms, we may have captured only subsyndromal anxiety. Moreover, we examined only a few of the many possible combinations of depressionanxiety comorbidity ${ }^{35}$. Lastly, sample sizes in some categories (Table 1) are low, hence the results should be interpreted keeping that in mind. Nevertheless, the study sample in TATAMS is large and representative of three cohorts of youth transitioning from adolescence to young adulthood. This is a significant age group to study as early-onset of psychiatric disorders may occur during adolescence. Moreover, the measures to assess symptoms are clinical measures commonly used in research and clinical settings today with sound psychometric properties, high validity and reliability $^{33,34}$.

\section{CONCLUSIONS}

The study findings can have implications for intervention planning, as interventions need to address marijuana and nicotine use in tobacco products and address anxiety and depression. The interventions may include promoting alternate coping resources and/or support (e.g. counseling, motivational interviewing) for depression and anxiety, to replace possible selfmedication with tobacco and/or marijuana. Given the increasing attention on anhedonia in smoking research, further examination can give an insight into whether anhedonia is predictive of poor cessation outcomes.

\section{REFERENCES}

1. Results from the 2017 National Survey on Drug Use and Health: Detailed Tables. U.S. Department of Health and Human Services; 2017. Accessed March 15, 2021. https:// www.samhsa.gov/data/sites/default/files/cbhsq-reports/ NSDUHDetailedTabs2017/NSDUHDetailedTabs2017. htm\#tab10-1A

2. Kaufman J, Charney D. Comorbidity of mood and anxiety disorders. Depress Anxiety. 2000;12(S1):6976. doi:10.1002/1520-6394(2000)12:1+<69::AIDDA9>3.0.CO;2-K

3. Clark LA, Watson D. Tripartite model of anxiety and depression: Psychometric evidence and taxonomic implications. J Abnorm Psychol. 1991;100(3):316-336. 
doi:10.1037//0021-843x.100.3.316

4. Wittchen HU, Kessler RC, Pfister H, Lieb M. Why do people with anxiety disorders become depressed? A prospective-longitudinal community study. Acta Psychiatr Scand Suppl. 2000;102(s406):14-23. doi:10.1111/j.0065-1591.2000.acp29-03.x

5. Pollack MH. Comorbid Anxiety and Depression. J Clin Psychiatry. 2005;66(suppl 8):22-29. Accessed November 29, 2021. https://www.psychiatrist.com/read-pdf/3379/

6. Garber J, Weersing VR. Comorbidity of Anxiety and Depression in Youth: Implications for Treatment and Prevention. Clin Psychol (New York). 2010;17(4):293306. doi:10.1111/j.1468-2850.2010.01221.x

7. Weinberger AH, Kashan RS, Shpigel DM, et al. Depression and cigarette smoking behavior: A critical review of population-based studies. Am J Drug Alcohol Abuse. 2017;43(4):416-431. doi:10.3109/00952990.2016.1171327

8. Fluharty M, Taylor AE, Grabski M, Munafò MR. The Association of Cigarette Smoking With Depression and Anxiety: A Systematic Review. Nicotine Tob Res. 2017;19(1):3-13. doi:10.1093/ntr/ntw140

9. Audrain-McGovern J, Leventhal AM, Strong DR. The Role of Depression in the Uptake and Maintenance of Cigarette Smoking. Int Rev Neurobiol. 2015;124:209243. doi:10.1016/bs.irn.2015.07.004

10. Bandiera FC, Loukas A, Li X, Wilkinson AV, Perry CL. Depressive Symptoms Predict Current E-Cigarette Use Among College Students in Texas. Nicotine Tob Res. 2017;19(9):1102-1106. doi:10.1093/ntr/ntx014

11. Bandiera FC, Loukas A, Wilkinson AV, Perry CL. Associations between tobacco and nicotine product use and depressive symptoms among college students in Texas. Addict Behav. 2016;63:19-22. doi:10.1016/j.addbeh.2016.06.024

12. Ameringer KJ, Leventhal AM. Applying the Tripartite Model of Anxiety and Depression to Cigarette Smoking: An Integrative Review. Nicotine Tob Res. 2010;12(12):1183-1194. doi:10.1093/ntr/ntq174

13. Marijuana Trends \& Statistics. National Institute on Drug Abuse. December 15, 2020. Accessed March 15, 2021. https://www.drugabuse.gov/drug-topics/marijuana/ marijuana-trends-statistics

14. Schauer GL, Rosenberry ZR, Peters EN. Marijuana and tobacco co-administration in blunts, spliffs, and mulled cigarettes: A systematic literature review. Addict Behav. 2017;64:200-211. doi:10.1016/j.addbeh.2016.09.001

15. Berg CJ, Payne J, Henriksen L, et al. Reasons for Marijuana and Tobacco Co-use Among Young Adults: A Mixed Methods Scale Development Study. Subst Use Misuse. 2018;53(3):357-369. doi:10.1080/10826084.2017.1327978

16. Schauer GL, Hall CD, Berg CJ, Donovan DM, Windle M, Kegler MC. Differences in the relationship of marijuana and tobacco by frequency of use: A qualitative study with adults aged 18-34 years. Psychol Addict Behav. 2016;30(3):406-414. doi:10.1037/adb0000172
17. Tucker JS, Rodriguez A, Dunbar MS, et al. Cannabis and Tobacco Use and Co-use: Trajectories and Correlates From Early Adolescence to Emerging Adulthood. Drug Alcohol Depend. 2019;204:107499. doi:10.1016/j.drugalcdep.2019.06.004

18. Cohn AM, Abudayyeh H, Perreras L, Peters EN. Patterns and correlates of the co-use of marijuana with any tobacco and individual tobacco products in young adults from Wave 2 of the PATH Study. Addict Behav. 2019;92:122127. doi:10.1016/j.addbeh.2018.12.025

19. Seaman EL, Stanton CA, Edwards KC, Halenar MJ. Use of tobacco products/devices for marijuana consumption and association with substance use problems among U.S. young adults (2015-2016). Addict Behav. 2020;102:106133. doi:10.1016/j.addbeh.2019.106133

20. Johnson KA, Bonn-Miller MO, Leyro TM, Zvolensky MJ.Anxious Arousal and Anhedonic Depression Symptoms and the Frequency of Current Marijuana Use: Testing the Mediating Role of Marijuana-Use Coping Motives Among Active Users. J Stud Alcohol Drugs. 2009;70(4):543-550. doi:10.15288/jsad.2009.70.543

21. Belujon P, Grace AA. Dopamine System Dysregulation in Major Depressive Disorders. Int J Neuropsychopharmacol. 2017;20(12):1036-1046. doi:10.1093/ijnp/pyx056

22. Dunlop BW, Nemeroff CB. The Role of Dopamine in the Pathophysiology of Depression. Arch Gen Psychiatry. 2007;64(3):327-337. doi:10.1001/archpsyc.64.3.327

23. de la Mora MP, Gallegos-Cari A, Arizmendi-García Y, Marcellino D, Fuxe K. Role of dopamine receptor mechanisms in the amygdaloid modulation of fear and anxiety: Structural and functional analysis. Prog Neurobiol. 2010;90(2):198-216. doi:10.1016/j.pneurobio.2009.10.010

24. Linsambarth S, Moraga-Amaro R, QuintanaDonoso D, Rojas S, Stehberg J. The Amygdale and Anxiety. In:Barbara F, ed. The Amygdala: Where Emotions Shape Perception, Learning and Memories. InTechOpen; 2017:139-171. Accessed November 29, 2021. https://pdfs.semanticscholar. org/a7da/b0f593e682ae5860c14cdc8fc5fa9fd1dcfe. pdf?_ga=2.252388681.1787947437.16393956391832160300.1602149247

25. Hjorth OR, Frick A, Gingnell M, et al. Expression and co-expression of serotonin and dopamine transporters in social anxiety disorder: a multitracer positron emission tomography study. Molecular psychiatry. 2021;26(8):3970-3979. doi:10.1038/s41380-019-0618-7

26. Rabin RA, George TP. A review of co-morbid tobacco and cannabis use disorders: possible mechanisms to explain high rates of co-use. Am J Addict. 2015;24(2):105-116. doi:10.1111/ajad.12186

27. Filbey FM, Gohel S, Prashad S, Biswal BB. Differential associations of combined vs. isolated cannabis and nicotine on brain resting state networks. Brain Struct Funct. 2018;223(7):3317-3326. doi:10.1007/s00429-018-1690-5 
28. Leventhal AM, Bae D, Kechter A, BarringtonTrimis J. Psychiatric comorbidity in adolescent use and poly-use of combustible, vaporized, and edible cannabis products. J Psychiatr Res. 2020;124:91-98. doi:10.1016/j.jpsychires.2020.02.021

29. Bonn-Miller MO, Zvolensky MJ, Johnson KA. UniMorbid and Co-Occurring Marijuana and Tobacco Use: Examination of Concurrent Associations with Negative Mood States. J Addict Dis. 2010;29(1):68-77. doi:10.1080/10550880903435996

30. Bottorff JL, Johnson JL, Moffat BM, Mulvogue T. Relieforiented use of marijuana by teens. Subst Abuse Treat Prev Policy. 2009;4:7. doi:10.1186/1747-597X-4-7

31. Leventhal AM, Strong DR, Sussman S, et al. Psychiatric comorbidity in Adolescent Electronic and Conventional Cigarette Use. J Psychiatr Res. 2016;73:71-78. doi:10.1016/j.jpsychires.2015.11.008

32. Pérez A, Harrell MB, Malkani RI, et al. Texas Adolescent Tobacco and Marketing Surveillance System's Design. Tob Regul Sci. 2017;3(2):151-167. doi:10.18001/TRS.3.2.3

33. Kroenke K, Spitzer RL. The PHQ-9: a new depression diagnostic and severity measure. Psychiatric annals. 2002;32(9):509-515. doi:10.3928/0048-5713-20020901-06

34. Spitzer RL, Kroenke K, Williams JB, Löwe B. A Brief Measure for Assessing Generalized Anxiety Disorder: The GAD-7. Arch Intern Med. 2006;166(10):1092-1097. doi:10.1001/archinte.166.10.1092

35. Hirschfeld RMA. The Comorbidity of Major Depression and Anxiety Disorders: Recognition and Management in Primary Care. Prim Care Companion J Clin Psychiatry. 2001;3(6):244-254. doi:10.4088/pcc.v03n0609

\section{CONFLICTS OF INTEREST}

The authors have each completed and submitted an ICMJE Form for Disclosure of Potential Conflicts of Interest. The authors declare that they have no competing interests, financial or otherwise, related to the current work. M. B. Harrell and S. Clendennen report being consultants in litigation involving the vaping industry.

\section{FUNDING}

This research was supported by grant (R01-CA239097) from the National Cancer Institute. The funding body had no role in the design and conduct of the study. The content is solely the responsibility of the authors and does not necessarily represent the official views of the NIH (NCI).

\section{ETHICAL APPROVAL AND INFORMED CONSENT}

The TATAMS human subject's methods were approved by the University of Texas Health Science Center at Houston Institutional Review Board (HSC$\mathrm{SPH}-13-0377$ ). Active consent from parents/guardians and assent from students were obtained for all surveys.

\section{DATA AVAILABILITY}

The data supporting this research are available from M. B. Harrell on reasonable request.

PROVENANCE AND PEER REVIEW

Not commissioned; externally peer reviewed. 